\title{
论 文
}

\section{红荧烯客体分子掺入 $\mathrm{Alq}_{3}$ 和 CBP主体的系间䇀越的 反常温度效应}

\author{
汤仙童, 许静, 邓金秋, 潘䜭亨, 胡叶倩, 熊祖洪, 陈晓莉 ${ }^{*}$ \\ 西南大学物理科学与技术学院, 重庆 400715 \\ *联系人, E-mail: xlchen@swu.edu.cn \\ 收稿日期: 2018-03-29; 接受日期: 2018-04-23; 网络出版日期: 2018-08-03 \\ 国家自然科学基金(编号: 11374242)资助项目
}

\begin{abstract}
摘要 为了研究基于红荧烯(Rubrene)发光器件的微观过程, 本文分别选用 $\mathrm{CBP}$ 和 $\mathrm{Alq}_{3}$ 作为主体材料, 制备了两种 红荧烯掺杂器件, 并在20-300 K温度范围测量了器件的电致发光磁效应(Magneto-Electroluminescence, MEL). 实 验发现器件的MEL曲线是由系间窝越(Intersystem Crossing, ISC)过程占主导的低场效应和三重态激子湮灭(Triplet-triplet Annihilation, TTA)过程占主导的高场效应两部分组成. 与未掺杂的常规有机发光二极管相比，同一温 度下, 这3种器件的低场效应呈现出相同的变化趋势, 不同温度下却表现出截然相反的变化规律. 即: 常规有机发 光器件中的低场效应表现为 ISC过程随着温度的降低而减弱，而红荧烯掺入 $\mathrm{Alq}_{3}$ 和 $\mathrm{CBP}$ 的器件的低场效应则表现 为 ISC过程随温度的降低而增强. 通过分析器件的能级结构、主体发射谱和客体吸收谱可知, 红荧烯掺入 $\mathrm{Alq}_{3}$ 和 CBP的器件的微观机制包括载流子陷阱效应和Förster能量转移过程, 其中载流子陷阱效应主要影响MEL的高场 效应且基本不受温度变化的影响. 因此, ISC过程的反常温度效应则是由于Förster能量转移过程在低温时被抑制, 导致主体材料极化子对数量的增加和极化子对间的ISC作用增强. 本研究有助于深入理解基于红荧烯发光器件微 观机制的演化过程.
\end{abstract}

关键词红荧烯, 电致发光磁效应, 系间鼌越, 载流子陷阱, Förster能量转移

PACS: 72.80.Le, 71.35.-y, 78.60.Fi, 78.55.Kz

\section{1 引言}

有机半导体红苂烯 $(5,6,11,12$-Tetraphenylnaphthacene, Rubrene)材料因具有较高的空穴迁移率 ${ }^{[1]}$ 、较长 的激子扩散长度 ${ }^{[2]}$ 、较强的单重态激子分裂能力 ${ }^{[3]}$ 以 及在柔性电子设备中的运用 ${ }^{[4]}$, 科学界已利用稳态、 瞬态、磁效应等方法对基于rubrene的薄膜和器件中 的光-电-磁学性质进行了大量研究 ${ }^{[5-13]}$. 其中, 电致发 光磁效应(Magneto-Electroluminescence, MEL) 已被作 为一种无损的指纹式工具来分析有机发光二极管中复 杂的自旋相关过程, 包括系间窝越(Intersystem Cross-

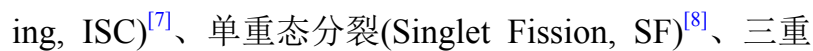

引用格式: 汤仙童, 许静, 邓金秋, 等. 红苂烯客体分子掺入 $\mathrm{Alq}_{3}$ 和 CBP主体的系间窝越的反常温度效应. 中国科学: 物理学 力学 天文学, 2018, 48: 117001 Tang X T, Xu J, Deng J Q, et al. Abnormal temperature dependent behaviors of intersystem crossing from rubrene guest dopant with Alq ${ }_{3}$ and $\mathrm{CBP}$ hosts (in Chinese). Sci Sin-Phys Mech Astron, 2018, 48: 117001, doi: 10.1360/SSPMA2018-00088 
态湮灭(Triplet-Triplet Annihilation, TTA) ${ }^{[0]}$ 等. 例如, Chen等人 ${ }^{[10]}$ 通过分析具有双功能的rubrene/ $\mathrm{C}_{60}$ 器件的 MEL，发现器件的电致发光是源于TTA过程而不是界 面俄歇复合形成的单重态激子. 最近，我们通过分析 注入较大电流条件下器件的MEL线型变化，指出SF占 主导的红苂烯器件中还存在ISC和TTA过程, 且这两种 过程产生的延迟苂光导致红荧烯器件中没有明显的效 率滚降现象 ${ }^{[11]}$. 然而, 当发光层为纯的红苂烯薄膜时, 通常器件中会发生明显的激子淬灭现象. 因此为了提 高器件的发光强度、效率以及稳定性，具有较低电离 势的rubrene常常作为掺杂剂被掺杂到主体材料中．例 如：Zhang等人 ${ }^{[12]}$ 探究了将Rubrene作为客体材料掺杂 到N,N'-diphenyl-N,N'-bis(3-methylphenyl)-1,1'-biphenyl-4,4'diamine (TPD)中对电致发光器件效率和稳定 性的影响; Di等人 ${ }^{[13]}$ 将rubrene掺杂到poly(9-vinylcarbazole) (PVK)中实现了聚合物发光二极管中高效地利 用三重态激子.

据文献报道 ${ }^{[13]}$ ，掺杂体系中主体的单重态能量 $\left(E_{\mathrm{S}}\right)$ 和三重态能量 $\left(E_{\mathrm{T}}\right)$ 要高于客体的 $E_{\mathrm{S}}$ 和 $E_{\mathrm{T}}$ 才能允许 发生高效的主客体能量转移过程且确保客体较长的三 重态激子寿命. 为了探究红苂烯掺杂体系中激发态间 的微观过程，本文选用4,4'-N,N'-dicarbazolebiphenyl (CBP) 和tris-8-hydroxyquinoline aluminum( $\left(\mathrm{Alq}_{3}\right)\left(E_{\mathrm{T}-\mathrm{CBP}}\right.$ $\left.\approx 2.56 \mathrm{eV}^{[14]}, E_{\mathrm{T}-\mathrm{Alq}_{3}} \approx 2.05 \mathrm{eV}^{[15]}, E_{\mathrm{T} \text {-rubrene }} \approx 1.14 \mathrm{eV}^{[16]}\right)$ 为主 体材料制备了两种红苂烯掺杂器件，并测量了不同温 度下这两种器件随电流变化的MEL曲线. 实验发现红 荧烯掺入 $\mathrm{Alq}_{3}$ 和 $\mathrm{CBP}$ 的器件的MEL曲线均在低磁场范 围表现为ISC过程占主导, 在高磁场范围表现为TTA过 程占主导. 与未掺杂的常规器件对比发现, 这两类器件 中的ISC过程呈现出一致的电流效应和完全相反的温 度效应, 即: 同一温度下, 这两类器件中ISC均随电流的 增大而减弱; 然而, 同一注入电流下, 常规器件中ISC随 着温度的降低而减弱, 这也与大多数掺杂体系中ISC随 温度的演变规律类似 ${ }^{[17,18]}$, 称之为正常温度效应; 红苂 烯掺入 $\mathrm{Alq}_{3}$ 和 $\mathrm{CBP}$ 的器件中观察到ISC随着温度的降 低而增强, 与常规器件中的演变规律相反, 称之为反常 温度效应. 通过分析红荧烯掺入 $\mathrm{Alq}_{3}$ 和 $\mathrm{CBP}$ 的器件的 能级结构和内部机制演化过程，发现载流子陷阱效应 和Förster能量转移共存的红苂烯掺杂器件中, 由于载 流子陷阱具有较弱的温度依赖关系且主要影响较高磁 场范围MEL线型，因此随着温度的降低，被抑制的
Förster能量转移过程使得主体材料中的极化子对数量 增多, 从而导致极化子对 (Polaron Pair, $\mathrm{PP}_{\mathrm{S}}$ 和 $\mathrm{PP}_{\mathrm{T}}$ ) 间的 ISC过程在低温的增强. 红荧烯掺入 $\mathrm{Alq}_{3}$ 和 $\mathrm{CBP}$ 的器件 中这种ISC过程的反常温度效应目前在文献中还未见 报道过. 本文通过对相关实验数据进行理论分析和细 致讨论，加深了对红苂烯掺杂体系中激发态演化过程 的认识.

\section{2 器件制备与测量}

本文以 $\mathrm{Alq}_{3}$ 为发光材料和主体材料分别制备了参 考器件 $\mathrm{A}$ 和器件 $\mathrm{B}$, 其中器件 $\mathrm{A}$ 的结构为 $\mathrm{ITO} / \operatorname{poly}(3,4-$ ethylenedioxythiophene):poly(styrenesulfonate)(PEDOT: PSS)/N,N'-bis(naphthalen-1-y)-N,N'-bis(phenyl)-benzidine(NPB) $\quad(60 \mathrm{~nm}) / \mathrm{Alq}_{3} \quad(100 \mathrm{~nm}) / \mathrm{LiF} \quad(1 \mathrm{~nm}) / \mathrm{Al}$ $(120 \mathrm{~nm})$, 器件 $\mathrm{B}$ 的结构为ITO/PEDOT:PSS/NPB $(60 \mathrm{~nm}) / \mathrm{Alq}_{3}: 3 \%$ rubrene $(40 \mathrm{~nm}) /$ Bathocuproine $(\mathrm{BCP})$ $(60 \mathrm{~nm}) / \mathrm{LiF}(1 \mathrm{~nm}) / \mathrm{Al}(120 \mathrm{~nm})$. 以CBP为主体材料制 备的红苂烯掺杂器件 $\mathrm{C}$ 的结构为: ITO/PEDOT:PSS/ NPB $(60 \mathrm{~nm}) / \mathrm{CBP}: x \%$ rubrene $(40 \mathrm{~nm}) / \mathrm{BCP}(60 \mathrm{~nm})$ $/ \mathrm{LiF}(1 \mathrm{~nm}) / \mathrm{Al}(120 \mathrm{~nm})$, 其中 $x$ 为掺杂浓度 (器件 $\mathrm{C}_{1}$ 为 低掺杂浓度 $3 \%$, 器件 $\mathrm{C}_{2}$ 为高掺杂浓度 $15 \%$. 由于其他 掺杂浓度与这两个掺杂浓度具有类似的变化规律，为 了使文章简洁，本文只呈现这两个掺杂浓度对应的测 量结果). 对于器件 $\mathrm{B}$ 和 $\mathrm{C}$ 而言, $\mathrm{NPB}$ 为空穴传输层, $\mathrm{Alq}_{3}$ (或 $\mathrm{CBP}$ ) 掺杂 rubrene为发光层, $\mathrm{BCP}$ 为电子传输层, ITO和 $\mathrm{Al}$ 分别为器件的阳极和阴极, 且器件的有效发 光面积为 $2 \mathrm{~mm} \times 3 \mathrm{~mm}$. 首先将有助于空穴注入的PEDOT: PSS水溶液旋涂于清洗干净的基片上,而后在超高 真空 $\left(<10^{-6} \mathrm{~Pa}\right)$ 蒸镀系统中进行热蒸发制备各功能层. 很多研究小组已报道 ${ }^{[5,19-22]}$, 经过本文中超高真空热蒸 发技术方式蒸镀的纯红苂烯薄膜或红苂烯掺杂薄膜通 常都呈现无定形形貌(Amorphous Morphology). 正如 Park等人 ${ }^{[23]}$ 所报道的，若对薄膜进行高于室温的退火 处理，薄膜则会出现岛状晶畴(Island-Like Crystalline Domains). 也就是，若在蒸镀薄膜时样品衬底高于室 温, 非晶和结晶的混合相则可能出现在红荧烯薄膜中. 为了消除非晶和结晶相的共存, 在沉积过程中, 本实验 将祄底保持在室温. 而后, 将制备好的样品保存在真空 室中 (优于 $\left.1 \times 10^{-2} \mathrm{~Pa}\right)$, 并在 $12 \mathrm{~h}$ 以内完成测量. 因此, 我 们制备的薄膜是没有晶筹的无定形薄膜. 在测量过程 
中, 先将制备好的器件置于电磁铁(Lakeshore：EM 647)磁极间的真空闭循环冷却系统(Janis: CCS-3050S) 的冷头上，再测量器件的MEL曲线、电流-发光-电压 特性曲线和光谱. 其中, 由Keithley 2400 万用表给器件 提供加载偏压, 由硅光电二极管测得并由Keithley 2000 输出器件的电致发光强度, 由SpectraPro-2300i光 栅光谱仪测量得到器件的光谱, 由计算机中的 Labview软件来控制整个测量系统，由Shimadzu UV-2600 仪器测量rubrene薄膜的吸收谱.

\section{3 实验结果与讨论}

\section{1 器件的基本特性}

器件 $\mathrm{B}$ 和器件 $\mathrm{C}_{1}$ 在室温下的 $I-V$ 特性曲线如图1(a) 所示, 呈现 $I \propto V^{\mathrm{m}}$ 的半导体特性, 器件结构如图1(a)的 插图所示. 图1(b)为室温下 $\mathrm{Alq}_{3}$ 和CBP的归一化光致发 光(Photoluminescence, PL)光谱, rubrene的归一化吸收 谱以及室温下器件 $\mathrm{A}$ 、器件 $\mathrm{B}$ 和器件 $\mathrm{C}_{1}$ 的归一化EL谱. 其中, $\mathrm{CBP}$ 和 $\mathrm{Alq}_{3}$ 的发光峰位分别约为 372 和 $531 \mathrm{~nm}$, rubrene薄膜的稳态吸收谱有 4 个峰值(436，465，496和 $531 \mathrm{~nm})$. 据Förster能量转移过程理论可知, 能量转换 半径 $R_{0}\left(R_{0}^{6}=\alpha \int_{0}^{\infty} F_{d}(v) \varepsilon_{a}(v) v^{-4} \mathrm{~d} v\right)$ 决定了主客体间的转 移效率，并且 $R_{0}$ 又与主客体分子的偶极矩方向 $(\alpha)$ 、主 体发射谱 $\left(F_{d}(v)\right)$ 和客体吸收谱 $\left(\varepsilon_{a}(v)\right)$ 的交叠等因素有 关 ${ }^{[24]}$. 如图1(b)中所示，器件B中主体材料 $\mathrm{Alq}_{3}$ 的发射 谱与客体rubrene的吸收谱具有较大部分的交叠, 器件
$\mathrm{C}_{1}$ 中 $\mathrm{CBP}$ 的发射谱也与rubrene吸收谱部分交叠. 由此 说明器件 $\mathrm{B}$ 和 $\mathrm{C}$ 中主客体间能够发生有效的Förster能 量转移过程且器件 $\mathrm{B}$ 中的能量转移效率更高. 此外, 从 图中还可以看出, 器件 $\mathrm{B}$ 和器件 $\mathrm{C}_{1}$ 的两个峰值均为 565 和605 nm, 与文献报道的rubrene发光峰位一致 ${ }^{[5,8,11]}$, 说 明了红苂烯掺杂器件中主体材料的苂光被完全淬灭, 同时也进一步证实了主客体间能量转移的有效性. 图1(c)为器件B在不同温度下的归一化EL谱. 据文献报 道 ${ }^{[5,19,25]}$, 565 和 $605 \mathrm{~nm}$ 处的苂光峰位分别对应 $0-0$ 转变 和0-1转变. 不同种类的分子聚集现象, 将导致光谱的 线型会随着温度的降低而发生两种不同的变化. 具体 来讲，J型聚集中，0-0峰会随着温度的降低而增强; $\mathrm{H}$ 型聚集中, 随着温度的降低, 0-0峰将失去其相对于 0-1 峰的相对强度(即0-0峰与 0-1峰之比会发生明显变化). 从图1(c)中可以看出, 0-0峰基本不随温度发生改变, 00 峰与0-1峰之比也未发生明显变化， $20 \mathrm{~K}$ 时，0-1峰的 略微缩小与较长的电子失相一致(Longer Electronic Dephasing Time $)^{[25]}$. 因此，在本文 $\mathrm{Alq}_{3}$ 掺杂 rubrene薄 膜中没有发生分子聚集. 此结论也与最近Tian等人 ${ }^{[19]}$ 报道的通过测量 $\mathrm{Alq}_{3}$ 掺杂rubrene薄膜的XRD图和随温 度变化的光谱图得出的结论一致. 此外, 类似的现象也 在器件 $\mathrm{C}$ 随温度变化的光谱中被观测到。

\section{2 温度和电流对器件MEL曲线的影响}

图2给出了器件 $\mathrm{A}$, 器件 $\mathrm{B}$ 和器件 $\mathrm{C}_{1}$ 在室温 $(300 \mathrm{~K})$ 和低温 $(20 \mathrm{~K})$ 时，不同注入电流下MEL随外加磁场变 化的关系. MEL定义为：MEL $=\Delta E L / E L=[E L(B)-E L$
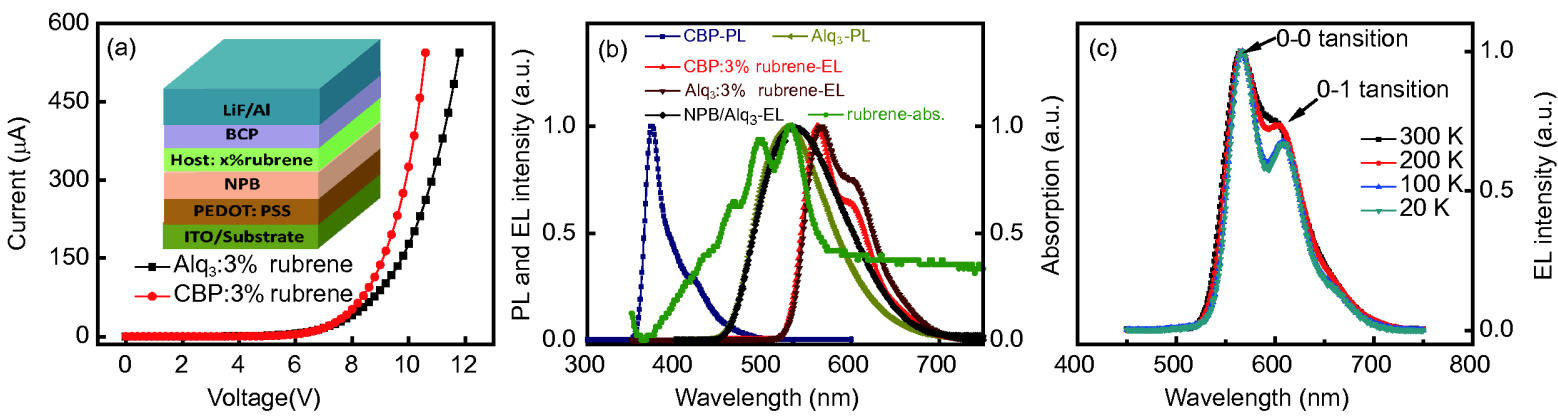

图 1 (网络版彩图) 器件的 $I-V$ 曲线、结构图、EL谱、材料的PL谱和吸收谱. (a) 室温下器件B和器件 $\mathrm{C}_{1}$ 的 $I-V$ 曲线, 插图为器 件的结构图; (b) 室温下 $\mathrm{Alq}_{3}$ 和CBP薄膜的归一化PL谱, rubrene薄膜的归一化吸收谱, 以及器件A、器件 $\mathrm{B}$ 和器件 $\mathrm{C}_{1}$ 的归一化EL 谱; (c) 器件B在不同温度下的归一化EL谱

Figure 1 (Color online) The $I-V$ curves, structure schematic, EL spectra of devices, normalized PL spectra and absorption spectra of materials. (a) The $I-V$ curves of devices B and $\mathrm{C}_{1}$, the inset is the structure schematic of device; (b) the normalized PL spectra of Alq $\mathrm{q}_{3}$ and CBP films, the normalized EL spectra of devices $\mathrm{A}, \mathrm{B}$, and $\mathrm{C}_{1}$, as well as the normalized absorption spectrum of rubrene film; (c) the temperature-dependent normalized EL spectra of device B. 

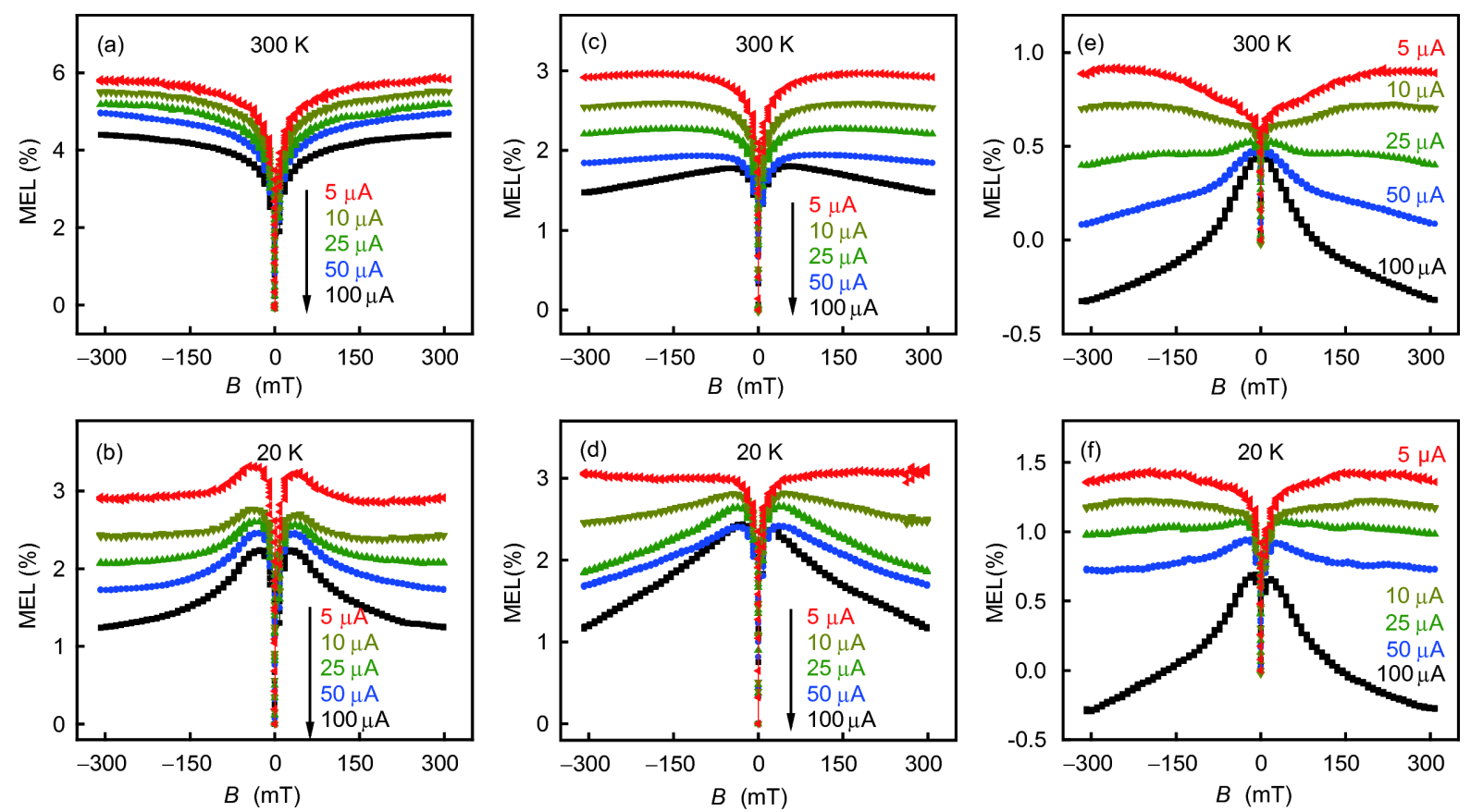

图 2 (网络版彩图) 温度为 $300 \mathrm{~K}$ 和 $20 \mathrm{~K}$ 时, 器件 $\mathrm{A}$, 器件 $\mathrm{B}$ 和器件 $\mathrm{C}_{1}$ 中随注入电流变化的MEL曲线. (a), (b) 器件 $\mathrm{A} ;$ (c), (d) 器件 $\mathrm{B}$; (e), (f) 器件 $\mathrm{C}_{1}$

Figure 2 (Color online) Current-dependent MEL curves of devices A, B, and $\mathrm{C}_{1}$ at $300 \mathrm{~K}$ and $20 \mathrm{~K}$. (a), (b) Device A; (c), (d) device B; (e), (f) device $\mathrm{C}_{1}$.

$(0)] \times 100 \% / \operatorname{EL}(0) ，$ 其中 $\operatorname{EL}(B)$ 和 $\operatorname{EL}(0)$ 分别表示有无磁 场时器件的电致发光强度. 从图2(a)和(b)中可以看出, 无论温度为 $300 \mathrm{~K}$ 还是 $20 \mathrm{~K}$, 常规器件 $\mathrm{A}$ (参考器件)的 MEL随磁场的增加可以分为两部分：在低磁场范围 $(|B|<27 \mathrm{mT}$ ) 快速增加(即低场效应，Low Filed Effect, LFE); 在高磁场范围 $(27 \mathrm{mT}<|B|<300 \mathrm{mT})$ 基本不变或 是缓慢减小(即高场效应, High Field Effect, HFE). 据文 献报道 ${ }^{[9,18]}$, 器件A 室温的MEL线型表现为典型的ISC 过程决定的指纹式响应曲线，低温的MEL线型则是由 低场的ISC过程和高场的TTA过程共同作用决定. 对于 $\mathrm{Alq}_{3}$ 掺杂rubrene的器件B而言, 如图2(c)和(d)所示, 温 度为 $300 \mathrm{~K}$ 时，注入电流 5-50 $\mu \mathrm{A}$ 范围内的MEL曲线与 器件 $A$ 室温的MEL曲线(见图2(a)) 具有类似的MEL线 型， $100 \mu \mathrm{A}$ 观察到的高场下降则是由于TTA作用引起 的; 温度为 $20 \mathrm{~K}$ 时，仍旧表现为与器件 $\mathrm{A}$ 低温下 (见 图2(b))类似的MEL线型，即随着注入电流的增加，低 场的ISC作用减弱与高场的TTA作用增强. 另外, CBP 掺杂rubrene的器件 $\mathrm{C}_{1}$ 对应的不同注入电流下的MEL 曲线如图2(e)和(f). 从图2(e)中可以看出, 室温下, 器件 $\mathrm{C}_{1}$ 与器件 $\mathrm{A}$ 和 $\mathrm{B}$ 相比具有类似的低场效应, 但在高磁场
范围内, 器件 $\mathrm{C}_{1}$ 的MEL 由小电流时的增加(即 SF过程的 体现)转变为大电流时的减小(即TTA过程的体现); 低 温下(见图2(f)), 器件 $\mathrm{C}_{1}$ 则表现出与器件 $\mathrm{A}$ 和 $\mathrm{B}$ 低温类 似的变化.

为了进一步研究温度对于器件中各激发态间相互 作用的影响, 以 $50 \mu \mathrm{A}$ 为例, 不同器件随温度改变的 MEL曲线如图3所示. 从图3(a)中可以清楚地看到, 当 温度从 $300 \mathrm{~K}$ 逐渐降为 $20 \mathrm{~K}$ 时, 随着磁场的增加, 器件 $\mathrm{A}$ 的低场范围表现为MEL的增幅逐渐减小, MEL的高 场也由室温的基本不变演变为低温的逐渐降低. 这与 之前文献报道的常规器件随温度的演化规律一致 ${ }^{[9,18]}$, 即低场效应反映出了ISC过程随温度的降低而减弱, 高 场效应反映出了 TTA过程随温度的降低而增强. 器件 A中ISC随温度的降低而减小的演化规律可以被称为 正常的温度效应. 器件 $\mathrm{B}$ 和器件 $\mathrm{C}_{1}$ 中 MEL随温度的演 化分别如图3(b)和(c)所示, 从图中可以看出, 随着温度 的降低, 高场效应均表现出与器件A类似高场的变化, 即TTA过程随着温度的降低而增强; 低场效应则均表 现出ISC随着温度的降低而增强, 此变化规律与器件 $\mathrm{A}$ 中ISC的演化规律(见图3(a))截然相反, 称之为反常温 

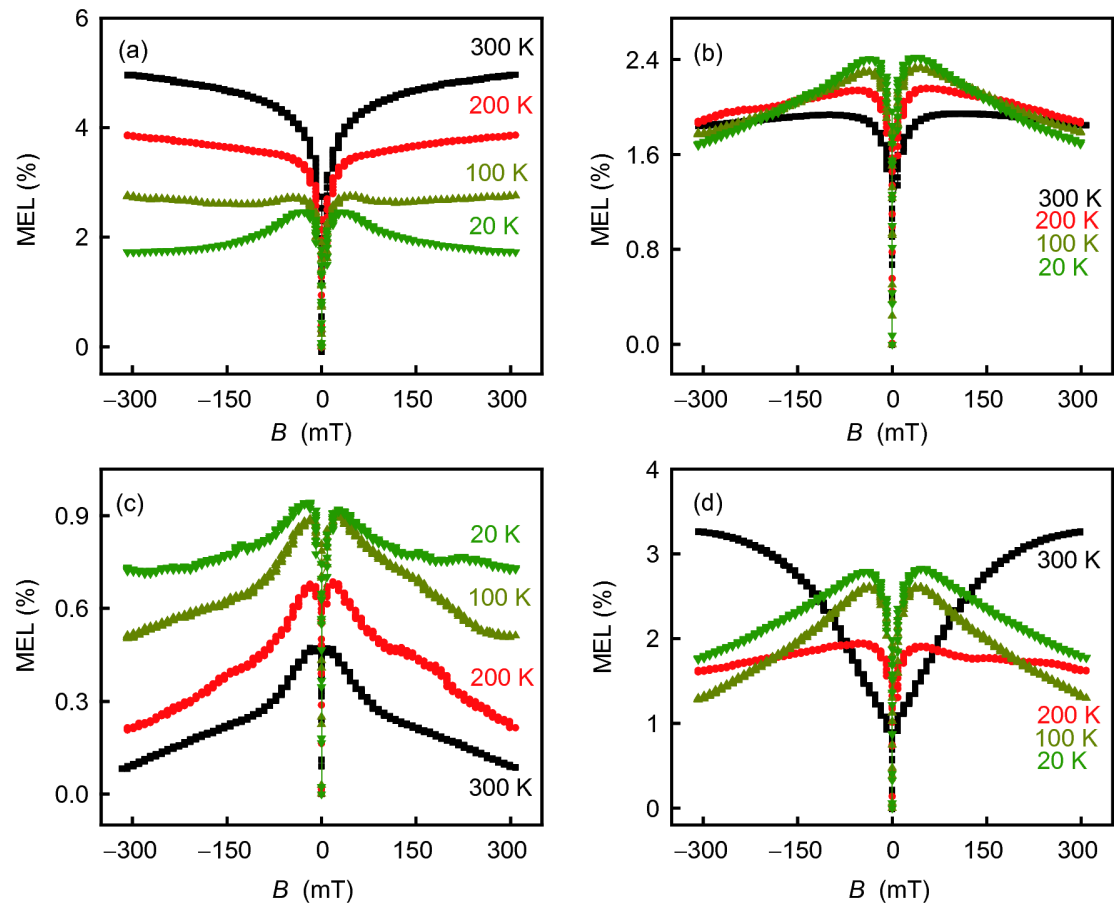

图 3 (网络版彩图) 注入电流为 $50 \mu \mathrm{A}$ 时器件在不同温度下的MEL曲线. (a) 器件A; (b) 器件B; (c) 器件 $\mathrm{C}_{1} ;$ (d) 器件 $\mathrm{C}_{2}$ Figure 3 (Color online) Temperature-dependent MEL curves of devices at $50 \mu \mathrm{A}$. (a) Device A; (b) device B; (c) device $\mathrm{C}_{1}$; (d) device $\mathrm{C}_{2}$.

度效应. 将CBP中rubrene的掺杂浓度增大为 $15 \%$ 得到 器件 $\mathrm{C}_{2}$, 其温度依赖关系如图3(d) 所示, 从图中可以观 察到, 由于rubrene的掺杂浓度变高, 其内部的单重态激 子分裂作用增强，导致室温HFE的MEL表现出随着磁 场的增加而增加(也就是更强的SF线型特征)，随着温 度的降低，TTA作用的增强导致了MEL出现高场下降， 但是LFE中ISC仍旧呈现反常温度效应.

为了定量比较不同器件中低场效应出现的ISC过 程的强弱，将低场磁效应值定义为: $\mathrm{MEL}_{\mathrm{LFE}}=\mathrm{MEL}(27$ $\mathrm{mT})-\operatorname{MEL}(0 \mathrm{mT})$, 并根据图2和3中所示的随注入电 流和温度变化的MEL曲线, 得到不同器件随注入电流 和温度的变化关系(如图4所示). 从图4(a)可以明显看 出, 无论是室温还是低温, 所有器件的 $\mathrm{MEL}_{\mathrm{LFE}}$ 都会随 着注入电流的增加而减小，也就是ISC过程随着电流 增加而减弱. 其中, 室温下器件A的ISC过程受电流调 控最明显. 从图4(b) 则可以看出, 红苂烯掺杂器件(即器 件 $\mathrm{B} 、$ 器件 $\mathrm{C}_{1}$ 和器件 $\mathrm{C}_{2}$ )均明显表现出与常规参考器件 (即器件 A) 完全相反的ISC演化规律，即随着温度从 $300 \mathrm{~K}$ 降为 $20 \mathrm{~K}$, 器件 $\mathrm{A} 由 3.7 \%$ 减小到 $2.4 \%$, 器件 $\mathrm{B}$ 、 器件 $\mathrm{C}_{1}$ 和器件 $\mathrm{C}_{2}$ 则分别增大了 $0.6 \%, 0.5 \%$ 和 $1.4 \%$. 综 上所述，这些器件中ISC过程产生的低场效应具有相
似的电流依赖关系，即ISC随着注入电流的增加而减 弱. 这种依赖电流变化的关系也与一般掺杂器件中 ISC随电流的演化规律也相似 ${ }^{[17,18,24]}$. 然而，红荧烯掺 入 $\mathrm{Alq}_{3}$ 和 CBP 的器件中出现的这种ISC过程的反常温 度效应在文献中还未见报道过．因此，本文将在第3.3 节细致分析各器件内部微观机制的演化以及ISC过程 反常温度效应产生的原因.

\section{3 分析与讨论}

器件 $\mathrm{A}$ 的能级结构如图5(a)所示，从图中可以看 出, NPB分子的 $\mathrm{HOMO}$ 能级 $(\sim 5.4 \mathrm{eV})$ 和 $\mathrm{Alq}_{3}$ 的 $\mathrm{HOMO}$ 能级 $(\sim 5.8 \mathrm{eV})$ 间具有较低的势垒 $(\sim 0.4 \mathrm{eV})$, 同时它们 的LUMO能级差约为 $0.7 \mathrm{eV}$. 因此，从ITO阳极注入的 空穴与 $\mathrm{A} 1$ 阴极注入的电子能在 $\mathrm{Alq}_{3}$ 层中复合. 如图 5 (b)所示, 由自旋统计理论可知, $\mathrm{Alq}_{3}$ 层中的电子和空 穴复合后会形成占比为 $25 \%$ 的单重态极化子对(Singlet Polaron Pair, $\mathrm{PP}_{\mathrm{S}}$ ) 和 $75 \%$ 的三重态极化子对(Triplet Polaron Pair, $\left.\mathrm{PP}_{\mathrm{T}}\right)^{[26]}$, 并且 $\mathrm{PP}_{\mathrm{S}}$ 能够通过ISC过程转化为 $\mathrm{PP}_{\mathrm{T}}$. 由于外加磁场会使 $\mathrm{PP}_{\mathrm{T}}$ 发生分裂, $\mathrm{PP}_{\mathrm{S}}$ 到 $\mathrm{PP}_{\mathrm{T}}$ 的转 化减弱，使得 $\mathrm{S}_{1}$ 的数量增加且发光增强; 当磁场强度 超过超精细耦合强度后，发光将很快趋于饱和，这就 

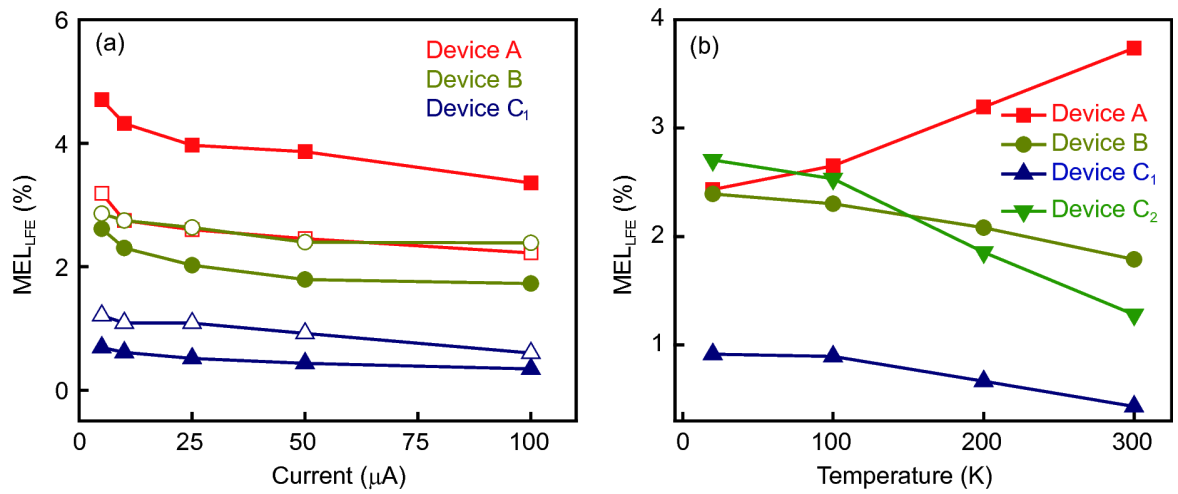

图 4 (网络版彩图)不同器件的低场效应随电流和温度的变化. (a) 器件 $\mathrm{A}$, 器件 $\mathrm{B}$ 和器件 $\mathrm{C}_{1}$ 在温度为 300 和 $20 \mathrm{~K}$ 时, 低场效应随 电流的变化, 其中实心点表示 $300 \mathrm{~K}$ 对应的变化, 空心点表示 $20 \mathrm{~K}$ 对应的变化; (b) 注入电流为 $50 \mu \mathrm{A}$ 时, 器件A, 器件B, 器件 $\mathrm{C}_{1}$ 和器件 $\mathrm{C}_{2}$ 的低场效应随温度的变化

Figure 4 (Color online) Low-filed effects of MEL curves varying with injected currents and temperature in different devices. (a) Current-dependent low-filed effects of devices $\mathrm{A}, \mathrm{B}$, and $\mathrm{C}_{1}$ at $300 \mathrm{~K}$ and $20 \mathrm{~K}$, solid dots denote the variation corresponding to $300 \mathrm{~K}$, hollow dots represent the variation corresponding to $20 \mathrm{~K}$; (b) temperature-dependent low-field effects of devices $\mathrm{A}, \mathrm{B}, \mathrm{C}_{1}$ and $\mathrm{C}_{2}$ at $50 \mu \mathrm{A}$.
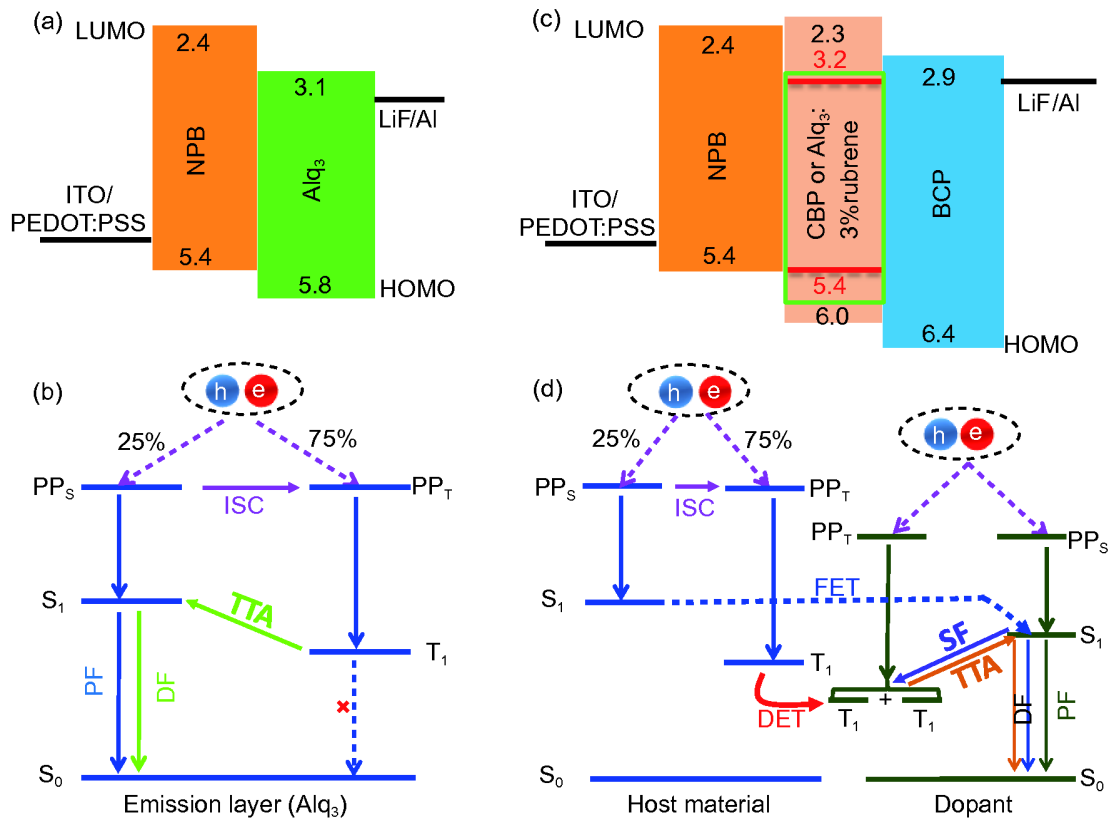

图 5 (网络版彩图) 器件中的能级结构和内部机制作用示意图. (a) 器件A的能级结构图; (b) 器件A中发光层内部机制的作用 示意图; (c) 器件 $\mathrm{B}$ 和器件 $\mathrm{C}_{1}$ 的能级结构图; (d) 器件 $\mathrm{B}$ 和器件 $\mathrm{C}_{1}$ 中掺杂层内部机制的作用示意图

Figure 5 (Color online) Energy diagrams and microscopic processes of devices. (a) The energy diagram of device A; (b) the schematic of microscopic process in device $\mathrm{A}$; (c) the energy diagram of device $\mathrm{B}$ and $\mathrm{C}_{1}$; (d) the schematic of energy transfer and microscopic process in device $\mathrm{B}$ and $\mathrm{C}_{1}$.

是ISC过程 $\left(\mathrm{PP}_{\mathrm{S}} \rightarrow \mathrm{PP}_{\mathrm{T}}\right)$ 对应的MEL线型(见图2(a)). 此 外, 由于库仑相互作用, $\mathrm{PP}_{\mathrm{S}}$ 和 $\mathrm{PP}_{\mathrm{T}}$ 也会进一步演化形 成对应的单重态激子(Singlet Exciton, $\mathrm{S}_{1}$ ) 和三重态激 子(Triplet Exciton, $\mathrm{T}_{1}$ ). $\mathrm{S}_{1}$ 能够直接辐射退激发射荧光 (Prompt Fluorescence, PF). $\mathrm{T}_{1}$ 本身由于自旋禁阻不能
直接辐射发光, 但两个 $T_{1}$ 可以发生 $T T A$ 过程 $\left(T_{1}+T_{1} \rightarrow S_{1}\right.$ $+\mathrm{S}_{0}, \mathrm{~S}_{0}$ 为 $\mathrm{Alq}_{3}$ 分子的基态)产生 $\mathrm{S}_{1}$, 从而向外辐射延迟 荧光(Delayed Fluorescence, DF). TTA过程对应的MEL 曲线特征通常表现为: 在较小磁场范围 $(|B|<25 \mathrm{mT})$, MEL随着磁场的增加而小幅增加, 在较大磁场范围 $(|B|$ 
$>25 \mathrm{mT}$ ), MEL随着磁场的增加而减小 ${ }^{[27]}$. 在常规激子 型发光器件中(也就是器件A), 室温下由于热声子的辅 助作用导致 $\mathrm{T}_{1}$ 寿命较短, 通常不能发生TTA过程, 若是 注入极大电流，增加 $\mathrm{T}_{1}$ 的浓度， TTA才可能在室温发 生 ${ }^{[28]}$. 随着温度的降低, 热声子的扰动减弱, $\mathrm{T}_{1}$ 寿命增 强, TTA过程也会增强, 如图2(b)所示.

对于本文的红苂烯掺杂体系而言，器件的能级结 构图如图5(c)所示, 从图中可以看出, 掺杂剂rubrene的 HOMO能级高于主体材料的HOMO能级，其LUMO能 级低于主体材料的LUMO能级，因此rubrene分子中能 够有效地形成载流子陷阱。此外，据第3.1节的分析可 知, 主客体间还存在有效的能量转移过程. 因此, 在红 荧烯掺杂器件中载流子陷阱效应与Förster能量转移过 程共同存在．红苂烯掺杂体系中存在的这两种不同的 微观机制将影响主客体上激子的形成和极化子对间的 相互作用，从而影响器件的MEL. 如图5(d)所示，注入 的部分载流子会直接在主体材料中形成 $\mathrm{PP}_{\mathrm{S}}$ 和 $\mathrm{PP}_{\mathrm{T}}$, 再 进一步形成主体中的 $\mathrm{S}_{1}$ 和 $\mathrm{T}_{1}$. 客体中的 $\mathrm{S}_{1}$ 和 $\mathrm{T}_{1}$ 则分别 是主体材料中 $\mathrm{S}_{1}$ 通过Förster能量转移(Förster Energy Transfer，FET)和主体材料中 $\mathrm{T}_{1}$ 通过Dexter能量转移 (Dexter Energy Transfer, DET)形成的 ${ }^{[29]}$. 此种情况下 器件中涉及的微观过程则不仅有客体rubrene分子间 的 $S F$ 过程 $\left(\mathrm{S}_{1}+\mathrm{S}_{0} \rightarrow \mathrm{T}_{1}+\mathrm{T}_{1}\right)$ 和TTA过程，还有主体材料 $\mathrm{CBP}$ 或 $\mathrm{Alq}_{3}$ 分子中极化子对间的ISC过程. 此外, $\mathrm{Luo}$ 等 人 ${ }^{[30]}$ 已证明在窄带隙材料掺杂系统中具有广泛存在的 能量捕获效应. 也就是说, 客体分子上的激子很难扩散 到主体材料，除非它们可以通过隧穿或其他激活方式 来克服主客体间的能量势垒 $\left(\Delta E_{\mathrm{HOMO}}\right.$ 和 $\left.\Delta E_{\mathrm{LUMO}}\right)$. 由于 这种载流子陷阱作用的存在，注入的另一部分载流子 还可以直接在rubrene分子中进行复合 ${ }^{[31]}$, 形成对应的 极化子对和激子，由于孤立的rubrene分子具有较高的 苂光量子产率(>98\%)但通过ISC过程转化成三重态的 产率小于 $1 \%^{[25]}$ ，以及rubrene中的ISC过程通常只能在 较大注入电流下 $(>1000 \mu \mathrm{A})$ 才能观察到 ${ }^{[11]}$. 因此本文 将不考虑rubrene分子间的ISC作用. 由于室温的激活 能 $(26 \mathrm{meV})$ 远小于分子克服 $\Delta E_{\mathrm{HOMO}}$ 和 $\Delta E_{\mathrm{LUMO}}$ 势垒的能 量(器件 $\mathrm{B}$ 中 $\Delta E_{\mathrm{HOMO}} \approx 0.4 \mathrm{eV}, \Delta E_{\mathrm{LUMO}} \approx 0.1 \mathrm{eV}$, 器件 $\mathrm{C}_{1}$ 中 $\left.\Delta E_{\mathrm{Hомо}} \approx 0.6 \mathrm{eV}, \Delta E_{\mathrm{LUMO}} \approx 0.9 \mathrm{eV}\right)$, 因此激子会被捕获在 客体材料中，减少了激子在热激发激子扩散期间通过 非辐射复合而淬灭的几率，同时也增强了三重态激子 寿命. 掺杂体系中增强的三重态寿命将导致较大的三
重态浓度, 因此TTA过程将在室温出现, 随着注入电流 的增加, 三重态浓度更大，导致更强的TTA过程，即更 强的高场效应(见图2(c)和(e))。随着温度的降低，由于 激活能 $k_{\mathrm{B}} \mathrm{T}$ 的值会更低, 激子更加难以克服 $\Delta E_{\mathrm{HO} \mathrm{MO}}$ 和 $\Delta E_{\mathrm{LUMO}}$ 势垒的能量，进一步减少了掺杂剂分子中的激 子发生淬灭的几率. 也就是说温度对载流子陷阱效应 影响较小, 表现为TTA随着温度的降低而增强, 但增加 的幅度变化不大(见图3(b)和(c)). 据以上分析可以认 为, 载流子陷阱效应的存在主要影响MEL的高场效应. 因此, 在以下分析产生低场效应的原因时, 则主要考虑 能量转移过程的作用.

同一温度条件下 (以 $300 \mathrm{~K}$ 为例), 如图2(c)和(e)所 示, 当器件中注入较小电流时, 主体材料 $\mathrm{Alq}_{3}$ 或 $\mathrm{CBP}$ 中 形成的 $\mathrm{PP}_{\mathrm{S}}$ 和 $\mathrm{PP} \mathrm{P}_{\mathrm{T}}$ 间会通过ISC过程发生相互转化. 且 $\mathrm{Alq}_{3}$ 或CBP中激子复合形成的 $\mathrm{S}_{1}$ 会通过Förster能量转 移过程将能量传递给客体, 并在客体上形成 $\mathrm{S}_{1}$. Förster 能量转移过程的强弱将直接影响主体中激子和极化子 对的数量. 随着注入电流的增加, 增强的电场将导致激 子和极化子对的解离增强，因此主体材料 $\mathrm{PP}_{\mathrm{S}}$ 和 $\mathrm{PP}_{\mathrm{T}}$ 间 的ISC过程将减弱, 如图4(a)所示. 然而, 随着温度的降 低, Förster能量转移过程被抑制, 使得主体材料中 $\mathrm{PP}_{\mathrm{S}}$ 数量增加, 那么 $\mathrm{PP}_{\mathrm{S}}$ 和 $\mathrm{PP}_{\mathrm{T}}$ 间的 ISC过程就会随着温度 的降低而增强，如图4(b)中所示，这就是红苂烯掺入 $\mathrm{Alq}_{3}$ 和CBP的器件中反常温度效应发生的原因.

\section{4 结论}

本文研究了红苂烯掺入 $\mathrm{Alq}_{3}$ 和 $\mathrm{CBP}$ 的器件中受电 流和温度调控的电致发光磁响应. 实验发现: 红苂烯掺 入 $\mathrm{Alq}_{3}$ 和CBP的器件和常规器件的MEL曲线均表现出 两段变化趋势, 即ISC过程占主导的低场效应和TTA调 控的高场效应. 其中, 红苂烯掺入 $\mathrm{Alq}_{3}$ 和 $\mathrm{CBP}$ 的器件的 低场效应具有正常的电流依赖关系以及反常的温度效 应，即ISC随着电流的增加而减弱，但会随着温度的降 低而增强. 通过分析器件的能级结构和内部机制发现, 红荧烯掺入 $\mathrm{Alq}_{3}$ 和 CBP 的器件的微观过程由Förster能 量转移和载流子陷阱作用共同决定. 具体来讲, 同一温 度下, 较小注入电流时, 主体材料中形成的极化子对间 通过ISC作用发生转化，当注入电流增大时，电场的增 加将导致激子和极化子发生解离, 从而主体间的ISC作 用减弱. 载流子陷阱作用将导致TTA过程出现在室温, 且注入电流增加导致三重态激子浓度增加，从而TTA 
过程增强. 同一注入电流条件下，随着温度的降低, Förster能量转移过程被抑制将增加主体中单重态极化 子数量, 从而增强主体中的ISC过程; 另外, 由于低温下 较低的激活能难以克服 $\Delta E_{\mathrm{HOMO}}$ 和 $\Delta E_{\mathrm{LUMO}}$ 势垒能量, 将
导致温度对载流子陷阱作用影响的较小，从而引起高 场的TTA作用在降温时有小幅增加. 本研究工作深入 分析了红苂烯掺杂器件中微观机制随电流和温度的演 化, 同时还丰富了有机磁效应的表现形式.

\section{参考文献}

1 Podzorov V, Menard E, Borissov A, et al. Intrinsic charge transport on the surface of organic semiconductors. Phys Rev Lett, 2004, 93: 086602

2 Najafov H, Lee B, Zhou Q, et al. Observation of long-range exciton diffusion in highly ordered organic semiconductors. Nat Mater, 2010, 9: 938943

3 Ma L, Zhang K, Kloc C, et al. Singlet fission in rubrene single crystal: Direct observation by femtosecond pump-probe spectroscopy. Phys Chem Chem Phys, 2012, 14: 8307-8312

4 Briseno A , Tseng R , Ling M M, et al. High-performance organic single-crystal transistors on flexible substrates. Adv Mater, 2006, 18: 23202324

5 Piland G B, Burdett J J, Kurunthu D, et al. Magnetic field effects on singlet fission and fluorescence decay dynamics in amorphous rubrene. J Phys Chem C, 2013, 117: 1224-1236

6 Tarasov V V, Zoriniants G E, Shushin A I, et al. The role of spin-lattice relaxation in magnetic field effects on the luminescence of amorphous and polycrystalline rubrene films. Chem Phys Lett, 1997, 267: 58-64

7 Kalinowski J, Cocchi M, Virgili D, et al. Magnetic field effects on emission and current in $\mathrm{Alq}_{3}$-based electroluminescent diodes. Chem Phys Lett, 2003, 380: 710-715

8 Bai J W, Chen P, Lei Y L, et al. Studying singlet fission and triplet fusion by magneto-electroluminescence method in singlet-triplet energyresonant organic light-emitting diodes. Org Electron, 2014, 15: 169-174

9 Liu R, Zhang Y, Lei Y L, et al. Magnetic field dependent triplet-triplet annihilation in $\mathrm{Alq}_{3}$-based organic light emitting diodes at different temperatures. J Appl Phys, 2009, 105: 093719

10 Chen Q, Jia W, Chen L, et al. Determining the origin of half-bandgap-voltage electroluminescence in bifunctional rubrene/ $\mathrm{C}_{60}$ devices. Sci Rep, 2016, 6: 25331

11 Tang $\mathrm{X}, \mathrm{Hu} \mathrm{Y}$, Jia $\mathrm{W}$, et al. Intersystem crossing and triplet fusion in singlet-fission-dominated rubrene-based OLEDs under high bias current. ACS Appl Mater Interfaces, 2018, 10: 1948-1956

12 Zhang Z L, Jiang X Y, Xu S H, et al. The effect of rubrene as a dopant on the efficiency and stability of organic thin film electroluminescent devices. J Phys D-Appl Phys, 1998, 31: 32-35

13 Di D, Yang L, Richter J M, et al. Efficient triplet exciton fusion in molecularly doped polymer light-emitting diodes. Adv Mater, 2017, 29: 1605987

14 Liu S, Li B, Zhang L, et al. Enhanced efficiency and reduced roll-off in nondoped phosphorescent organic light-emitting devices with triplet multiple quantum well structures. Appl Phys Lett, 2010, 97: 083304

15 Jia W, Chen Q, Chen L, et al. Molecular spacing modulated conversion of singlet fission to triplet fusion in rubrene-based organic light-emitting diodes at ambient temperature. J Phys Chem C, 2016, 120: 8380-8386

16 Zhang Y, Forrest S R. Triplets contribute to both an increase and loss in fluorescent yield in organic light emitting diodes. Phys Rev Lett, 2012, 108: 267404

17 Chen P, Lei Y L, Song Q L, et al. Magnetoelectroluminescence in tris (8-hydroxyquinolato) aluminum-based organic light-emitting diodes doped with fluorescent dyes. Appl Phys Lett, 2009, 95: 213304

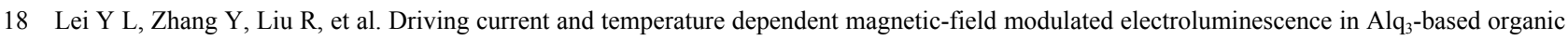
light emitting diode. Org Electron, 2009, 10: 889-894

19 Tian X, Zhou L, Chen X, et al. Nanoscale exponential distance dependence and electron-transfer model for intermolecular singlet exciton fission observed in rubrene-doped organic films. Org Electron, 2017, 50: 429-434

20 Li J, Chen Z, Zhang Q, et al. Temperature-dependent singlet exciton fission observed in amorphous rubrene films. Org Electron, 2015, 26: 213- 
217

21 Jankus V, Snedden E W, Bright D W, et al. Competition between polaron pair formation and singlet fission observed in amorphous rubrene films. Phys Rev B, 2013, 87: 224202

22 Seo J H, Park D S, Cho S W, et al. Buffer layer effect on the structural and electrical properties of rubrene-based organic thin-film transistors. Appl Phys Lett, 2006, 89: 163505

23 Park S W, Choi J M, Lee K H, et al. Amorphous-to-crystalline phase transformation of thin film rubrene. J Phys Chem B, 2010, 114: 5661-5665

24 Kan M, Chen P, Cao S Q, et al. Triplet-triplet annihilation process in organic light-emitting diodes doped with fluorescent dyes (in Chinese). Chin Sin Bull, 2010, 55: 1358-1364 [阙敏, 陈平, 曹绍谦, 等. 三重态激子在不同苂光染料掺杂体系中的湮灭过程. 科学通报, 2010, 55: 13581364]

25 Li J, Chen Z, Lei Y, et al. Competition between singlet exciton fission, radiation, and dissociation measured in rubrene-doped amorphous films. Synth Met, 2015, 207: 13-17

26 Desai P, Shakya P, Kreouzis T, et al. Magnetoresistance and efficiency measurements of Alq ${ }_{3}$-based OLEDs. Phys Rev B, 2007, 75: 094423

27 Yokoyama K, Wakikawa Y, Miura T, et al. Solvent viscosity effect on triplet-triplet pair in triplet fusion. J Phys Chem B, 2015, 119: 1590115908

28 Zhang Y, Liu R, Lei Y L, et al. Prompt fluorescence and delayed fluorescence in Alq ${ }_{3}$-based organic light-emitting diode (in Chinese). Sci SinPhys Mech Astron, 2010, 40: 416-424 [张勇, 刘荣, 雷衍连, 等. 基于 $\mathrm{Alq}_{3}$ 的有机发光二极管中的瞬时苂光与延迟菼光. 中国科学: 物理学 力 学 天文学, 2010, 40: 416-424]

29 Deng J, Jia W, Chen Y, et al. Guest concentration, bias current, and temperature-dependent sign inversion of magneto-electroluminescence in thermally activated delayed fluorescence devices. Sci Rep, 2017, 7: 44396

30 Luo Y, Aziz H, Klenkler R, et al. Temperature dependence of photoluminescence efficiency in doped and blended organic thin films. Chem Phys Lett, 2008, 458: 319-322

31 Ingram G L, Nguyen C, Lu Z H. Long-range energy transfer and singlet-exciton migration in working organic light-emitting diodes. Phys Rev Appl, 2016, 5: 064002 


\title{
Abnormal temperature dependent behaviors of intersystem crossing from rubrene guest dopant with $\mathrm{Alq}_{3}$ and CBP hosts
}

\author{
TANG XianTong, XU Jing, DENG JinQiu, PAN RuiHeng, HU YeQian, XIONG ZuHong \\ \& CHEN XiaoLi*
}

School of Physical Science and Technology, Southwest University, Chongqing 400715, China

\begin{abstract}
In order to study the microscopic process in rubrene-doped organic light emitting diodes, two kinds of devices were fabricated by utilizing the host materials of 4,4'-N,N'-dicarbazolebiphenyl (CBP) and tris-8-hydroxyquinoline aluminum $\left(\mathrm{Alq}_{3}\right)$, as well as the fluorescent dopant material 5,6,11,12-tetraphenylnaphthacene (rubrene). The magnetoelectroluminescence (MEL) curves were measured in the temperature range of $20 \mathrm{~K}$ to $300 \mathrm{~K}$. It was found that the MEL curves of rubrene dopant with $\mathrm{Alq}_{3}$ and CBP devices were composed of two parts, namely, the low field effect dominated by the intersystem crossing (ISC) process and the high field effect dominated by the triplet-triplet annihilation (TTA) process. Compared with the conventional un-doped device, the low field effects of these three devices exhibit the same change trend at the same temperature. At different temperatures, these devices show diametrically opposite changes. Specifically, the low field effect in un-doped device shows that the ISC process weakens with the decrease of the temperature, but the low field effects of rubrene dopant with $\mathrm{Alq}_{3}$ and $\mathrm{CBP}$ devices show that the ISC process increases with decreasing temperature. By analyzing the energy level structure of the device, the emission spectra of host materials and absorption spectra of guest material, it demonstrated that both the carrier trap effect and the Förster energy transfer process were included in the microscopic processes of rubrene dopant with $\mathrm{Alq}_{3}$ and $\mathrm{CBP}$ devices. The carrier trap effect mainly affects the high field effect of MEL and is basically independent of temperature changes. This anomalous temperature-dependent behavior of ISC process can be ascribed to the suppression of Förster energy transfer process at low temperatures, leading to an increase in the number of polaron pairs in the host material. Therefore, the ISC interaction between the polaron pairs will increase with decreasing temperature. Our work has a certain role in promoting the understanding for the evolution of microscopic mechanisms in rubrene doped devices.
\end{abstract}

rubrene, magneto-electroluminescence, intersystem crossing, carrier trap effect, Förster energy transfer

PACS: 72.80.Le, 71.35.-y, 78.60.Fi, 78.55.Kz

doi: 10.1360/SSPMA2018-00088 